\title{
A Construction of Two-Dimensional Quantum Chromodynamics
}

\author{
Sławomir Klimek ${ }^{1}$ and Witold Kondracki ${ }^{2}$ \\ 1 Department of Mathematical Methods of Physics, Warsaw University, PL-00-682 Warsaw, \\ Hoża 74, Poland \\ 2 Institute of Mathematics, Polish Academy of Sciences, PL-00-950 Warsaw, Śniadeckich 8, \\ Poland
}

\begin{abstract}
We present a sketch of the construction of the functional measure for the $S U(2)$ quantum chromodynamics with one generation of fermions in twodimensional space-time. The method is based on a detailed analysis of Wilson loops.
\end{abstract}

\section{Introduction}

In this paper we present a sketch of the construction of the functional measure for the simplest non-abelian gauge theory with fermions: the two-dimensional quantum chromodynamics. We shall concentrate on the main technical steps and estimates; for more details the reader is referred to [1].

Our methods are rather limited to the two-dimensional case since we use the solvability of the lattice pure gauge theory. However, the way we incorporate fermions into the theory can be, in principle, repeated in 3-dimensions. This may be important for further research since the interaction with fermions has been much less rigorously studied than with the Higgs fields [2], contrary to one's intuition that non-selfinteracting fermions should be easier to treat than self-interacting bosons. We speculate also on the implementation of the renormalization group ideas which look quite natural in our framework.

The paper consists of three parts. Section 1 contains the description of our methods and some preliminary constructions. Main results with sketches of proofs are given in Sect. 2. Finally in Appendix 1 one can find the necessary facts from measure theory and in Appendix 2 the proof of the basic estimate from lattice gauge theory.

\section{From Wilson Loops to Functional Measure}

In this section we collect miscellaneous facts about the orbit space, Wilson loops and some lattice gauge theory results and explain the main idea of our approach.

Let $\mathscr{A}$ be the space of gauge potentials (connections) on $\mathbb{R}^{2}$. These are one forms $A_{x} d x+A_{y} d y$ with coefficients taking values in a Lie algebra 5 of a compact 
Lie group $G$. For the sake of simplicity we shall focus our attention on $G=S U(2)$, but we would like to underline that our methods work for a larger class of compact groups. Let $\mathscr{G}$ be the group of local gauge transformations, i.e. the group of functions $\phi: \mathbb{R}^{2} \rightarrow G$.

$\mathscr{G}$ acts on $\mathscr{A}$ by the usual formula

$$
A \rightarrow \phi A \phi^{-1}+(d \phi) \phi^{-1} .
$$

We need the following convenient parametrization of the orbit space $\mathscr{A} / \mathscr{G}$ (based on axial gauge)

Lemma 1. a) In every orbit of $\mathscr{G}$ in $\mathscr{A}$ there exists a representative of the form

$$
A=A(x, y) d y ; \quad A(0, y)=0 .
$$

b) Two representatives of the form (1) differ by a constant gauge transformation, i.e.

$$
A_{1}(x, y)=g A_{2}(x, y) g^{-1}, \quad g \in G .
$$

Connections of form (1) can be parametrized by their curvatures

$$
F=\frac{\partial A}{\partial x} \text {. }
$$

Conversely, any function $F: \mathbb{R}^{2} \rightarrow \mathfrak{5}$ is the curvature of a connection of form (1) with

$$
A(x, y)=\int_{0}^{x} F(t, y) d t .
$$

Thus, there exists one to one correspondence between the orbit space $\mathscr{A} / \mathscr{G}$ and the space $B$ of functions on $\mathbb{R}^{2}$ with values in $(\mathfrak{5}$ (curvatures) or more exactly with the quotient $B / G$, where $G$ acts on $B$ by conjugation. Since $G$ is a compact group we shall not need to extract degrees of freedom connected with it. From now on we consider $B$ to be an orbit space where the functional measure should be defined [3]. We should only ascertain that the functional measure is defined invariantly under the residual $G$ symmetry. The specification of the class of functions in $B$ will be done in Sect. 2.

Of course one can rewrite the formal functional measure of the twodimensional Yang-Mills theory in terms of $B$. The corresponding FaddeevPopov determinant is constant, and one finds a gaussian measure which can be defined rigorously through its Fourier transform.

Instead, we propose a different procedure which is better suited, in our opinion, for the inclusion of fermions. Namely, we shall rather use the properties of the expectations of the Wilson loops $\langle W(C)\rangle$ than the Fourier transform $\left\langle e^{i\langle F, f\rangle}\right\rangle$ to prove an analogue of the Minlos theorem for Wilson loops, but as we explain below, will be useful in the definition of the regularized fermionic determinants. The expectation values of the Wilson loops will be taken from lattice calculations [4] and we shall also use lattice methods to regularize the fermionic determinants which on the lattice can be expressed by the random walk expansion in terms of Wilson loops. This is the basic reason why we take the Wilson loops as fundamental objects in defining the pure gauge theory functional measure. 


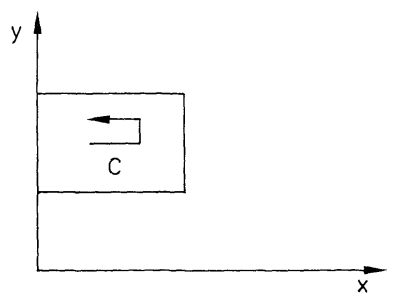

Fig. 1

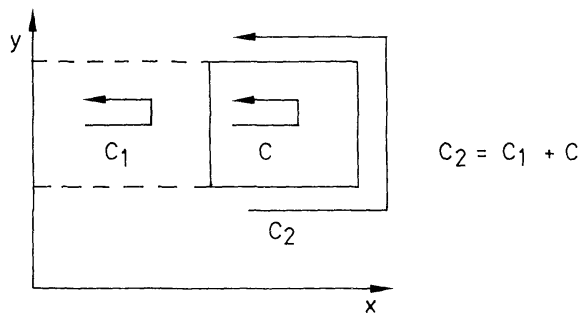

Since $F$ determines $A$ we can express the parallel transports of $A$ in terms of $F$ (in our gauge fixing). This is particularly simple for rectangles with one side on the $y$-axis, and more generally for rectangles with sides parallel to the coordinate axes. It is enough for our purposes to consider only such cases. We always choose the starting point for parallel transport to be the left, bottom corner of the rectangle. Appropriate formulas are the following (see Fig. 1):

$$
P_{c}(F)=T_{y} e^{i} \int_{S(C)} F \quad(\text { case } 1),
$$

where $S(C)$ is the area enclosed by $C$ and $T_{y}$ denotes path ordering in the $y$ direction

$$
P_{c}(F)=P_{c_{1}}(F)^{-1} \cdot P_{c_{2}}(F) \quad(\text { case } 2) \text {. }
$$

Wilson loops are, by definition, traces of parallel transports. For the case of interest, i.e. $G=S U(2)$, let $l=0, \frac{1}{2}, 1, \frac{3}{2}, \ldots$ label the irreducible representations and consider

$$
W^{l}(C)[F]=\chi_{l}\left(P_{c}(F)\right),
$$

where $\chi_{l}$ is the trace of the $l$-th representation. Then, from lattice gauge theory calculations, we know that the mean value of Wilson loops $W^{l}(C)$ with respect to the pure gauge theory functional measure is given by

$$
\left\langle W^{l}(C)\right\rangle=(2 l+1) e^{-l(l+1) S(C)} .
$$

Here we put for simplicity the coupling constant equal to one, for its actual value does not matter in our considerations. Moreover Wilson loops are on the lattice known to be independent random variables for loops $C$ with non-overlapping areas. Using formula (4) and the Fourier transformation on $G$ one can compute the integrals of more general functions on $B$,

$$
F \rightarrow f \cdot P_{C}(F),
$$

where $f: G \rightarrow \mathbb{C}^{1}$ is a continuous, class function on $G$. Writing

$$
f(g)=\sum_{l} a_{l} \chi_{l}(g) \quad \text { with } \quad a_{l}=\int f(g) \chi_{l}(g) d g
$$


one obtains

$$
\left\langle f \cdot P_{C}(F)\right\rangle=\int f \cdot P_{C}(F) d \mu(F)=\int f(g)\left(\sum_{l}(2 l+1) e^{-l(l+1) S(C)} \chi_{l}(g)\right) d g .
$$

The meaning of this formula is the following: (ignore for a moment that $f$ is a class function) transports of $\mu$ by means of $P_{C}$ are given by

$$
P_{C} \mu=\left(\sum_{l}(2 l+1) e^{-l(l+1) S(C)} \chi_{l}(g)\right) d g .
$$

The right-hand side of (6) is manifestly invariant under conjugation as it should be if $\mu$ is invariant under the residual $G$ symmetry. Thus (6) is compatible with the requirement that $\mu$ lives on $B / G$. The function in the parenthesis

$$
K(g, S(C))=\sum_{l}(2 l+1) e^{-l(l+1) S(C)} \chi_{l}(g)
$$

is the well-known heat kernel for the Laplace-Beltrami operator on $G$ [5]. For $t>0, K(g, t)$ is a smooth, positive class function on $G$ satisfying the equation

$$
\frac{\partial}{\partial t} K(g, t)=\Delta_{G} K(g, t) ; \quad K(g, 0)=\delta_{\text {id }}
$$

[in particular (6) really defines a measure]. Now the line of reasoning which we will pursue in detail in Sect. 2 goes as follows. Knowing the "projections" of $\mu$ by $P_{C}$ we will construct the adequate projective system of measures and use the Prokhorov theorem (see the appendix) to construct $\mu$. The crucial point is that $P_{C}$ are randomly independent for loops with non-overlapping areas. This makes the construction particularly simple. We shall prove (Theorem 1) that there exists only one measure $\mu$ on the (suitably defined) space $B$ satisfying (6). This measure should be identified with the functional measure for pure $S U(2)$ gauge theory.

Next, we include fermions into the model by the standard Matthews-Salam procedure. This procedure interprets the interaction with fermions as a kind of perturbation of pure gauge theory. From a technical point of view the MattewsSalam trick leads to the effective measure $\tilde{\mu}$ for gauge fields given by $d \tilde{\mu}=\operatorname{det} \not{ }_{A} d \mu$, where $\not_{A}$ is the Dirac operator (with a mass term) and $\operatorname{det} D_{A}$ is a gauge invariant quantity.

The determinant of the Dirac operator can be rigorously defined for the class of sufficiently regular connections. However general wisdom tells that $\mu$ and $\tilde{\mu}$ are actually orthogonal. This, in turn, means that $\operatorname{det} \not_{A}$ cannot be defined as a function on whole $B$ and a certain regularization procedure must be implemented. Let us consider the determinant of the $\varepsilon$-lattice Dirac operator in volume $V$ (for Wilson fermions in the fundamental representation). It is the (lattice) gauge invariant quantity depending polynomially on the coefficients, and thus can be expressed as a polynomial of lattice Wilson loops. To see this, recall that the determinant of the matrix $\not_{A}$ can be rewritten as a polynomial of $\operatorname{Tr}\left(\not_{A}\right)^{n}$, $n=1,2, \ldots,|V|$, and the last functions have a well-known polynomial expansion in terms of Wilson loops [6]. Consequently

$$
\operatorname{det}_{\varepsilon}^{v} \not D_{A}=\operatorname{polynomial}\left(\operatorname{Tr} P_{C}(A)\right)
$$


where the detailed form of the polynomial in (8) will not be crucial in our discussion. Since we consider fermions in the fundamental representation of $G, \mathrm{Tr}$ stands for the character of this representation.

Formula (8) is precisely what we need since the Wilson loops carry all the information about $\mu$ and consequently are to be defined on $B$. Now, we can regularize $\tilde{\mu}$ simply by putting

$$
d \tilde{\mu}_{\varepsilon}^{v}=\operatorname{det}_{\varepsilon}^{v} \not D_{A} d \mu .
$$

As a result of our construction of $\mu$, it will be a well defined measure on $B$. Cutoffs $\varepsilon, v$ can be next removed by studying the limit of measures $\tilde{\mu}_{\varepsilon}^{v}$ in a sufficiently weak topology on the space of measures on $B$. It is a classical problem in probability theory and our second main result (Theorem 2) states that the sequence $\tilde{\mu}_{\varepsilon}^{v}$ has indeed a convergent subsequence. This establishes the existence of (a candidate for) the functional measure for $(\mathrm{QCD})_{2}$.

\section{Outline of Proofs}

In this section we state our main results and outline their proofs. Let us first describe the space on which the functional measure is to be defined. Consider a strictly positive, self-adjoint and Hilbert-Schmidt (scalar) operator $K$ in $L^{2}\left(\mathbb{R}^{2}\right)$. Let $J=K^{2}$ and $J(x, y)$ denote the kernel of $J$,

$$
J f(x)=\int J(x, y) f(y) d y .
$$

Notice that $J$ is a strictly positive, self-adjoint and nuclear operator in $L^{2}\left(\mathbb{R}^{2}\right)$.

Moreover we shall require that the image of $K$ in $L^{2}\left(\mathbb{R}^{2}\right)$ contains a sufficiently large family of step functions, for example characteristic functions of elementary squares of the lattice $\frac{1}{2^{n}} \cdot Z^{2}$.

We define the Hilbert space $B$ as a completion of $L^{2}\left(\mathbb{R}^{2},(\mathfrak{G})\right.$ in the norm

$$
\|F\|^{2}=(F, J F)_{L^{2}\left(\mathbb{R}_{2}, \mathfrak{F}\right)}=(K F, K F)_{L^{2}\left(\mathbb{R}_{2} \cdot \mathfrak{G}\right)} .
$$

Following the discussion of Sect. 1 we consider $B$ to be the orbit space of the 2-dim. gauge-theory.

The definitions are motivated by the following consideration. The pure gauge theory functional measure is a gaussian measure with covariance equal to the scalar product of $L^{2}\left(\mathbb{R}^{2},(5)\right.$. By the usual argument related to the ProkhorovSazanov theorem, such a measure can be constructed on $B$ provided $J$ is selfadjoint and nuclear.

In electrodynamics the Wilson loop

$$
F \rightarrow e^{i \int_{s} F}
$$

is a continuous function of $B$ if and only if

$$
\chi_{s} \in \operatorname{Im} K \text {. }
$$

Thus, the above stated property of $\operatorname{Im} K$ guarantees the continuity of Wilson loops. We shall see that this remains true in the non-abelian case. Now we proceed to the 
definition of the parallel transports on $B$. Consider a rectangle $C$ with one side on the $y$-axis.

If $F \in C_{0}^{\infty}\left(\mathbb{R}^{2},(\mathfrak{5})\right.$ we put

$$
P_{C}(F)=T_{y} \exp i \iint_{S(C)} F \quad[\text { see }(2)] .
$$

The basic observation is the following:

Lemma 2. The map $P_{C}: C_{0}^{\infty}\left(\mathbb{R}^{2},(\mathfrak{5}) \rightarrow G\right.$ is uniformly continuous with respect to the norm (14).

Thus $\left\{P_{C}\right\}$ has a unique continuous and even uniformly continuous extension to the whole $B$ which will be also denoted by $P_{C}$. If $C$ is an arbitrary rectangle with sides parallel to coordinate axes we can define $P_{C}$ on $B$ with the help of formula (3).

Lemma 3. The family of maps $\left\{P_{C}\right\}$, where $C$ are rectangles with sides parallel to coordinate axes, separates points of $B$.

It is useful to consider the following topology on $B$ :

Definition. Wilson topology (W.T.) on $B$ is the weakest topology such that $P_{C}$ are still continuous.

Observe that W.T. is Haussdorff because of Lemma 3. W.T. is given by the set of nonlinear functions, however we expect it to coincide with the weak topology on $B$. One reason for this conjecture is the following fact:

Lemma 4. Closed balls in B with center at the origin are compact in W.T.

Now we are ready to state the first of our main results.

Theorem 1. There exists a unique probabilistic, Radon measure $\mu$ on $B$ such that

$$
P_{C} \mu=K(g, S(C)) d g,
$$

where $K(g, S(C))$ is the heat kernel of the Laplace-Beltrami operator on $G$ (see Sect. 1), $S(C)$ is an area enclosed by $C$ and $d g$ is the Haar measure on $G$.

b) $\left\{P_{C}\right\}$ are independent random variables for non-overlapping rectangles.

Idea of Proof. The proof requires some additional constructions related to lattice gauge theories. Consider the space

$$
\mathscr{G}_{\varepsilon}=\left\{g: \varepsilon Z^{2} \rightarrow G\right\}
$$

and equip $\mathscr{G}_{\varepsilon}$ with the Tichonoff topology. Thus $\mathscr{G}_{\varepsilon}$ is a compact space. We associate to any finite subset $V \subset \varepsilon Z^{2}$ the subspace $\mathscr{G}_{\varepsilon}^{V} \subset \mathscr{G}_{\varepsilon}$ by

$$
\mathscr{G}_{\varepsilon}^{V}=\left\{g \in \mathscr{G}_{\varepsilon}: \operatorname{supp} g \subset V\right\} .
$$

Notice that the family $\{V\}$ is directed by inclusion and

$$
\mathscr{G}_{\varepsilon}^{V}=\underbrace{G \times \ldots \times G,}_{|V| \text { times }}
$$

where $|V|$ is the number of points in $V$. Let $\mu_{\varepsilon}^{V}$ be the measure on $\mathscr{G}_{\varepsilon}^{V}$ given by the product of heat kernels:

$$
d \mu_{\varepsilon}^{V}=\prod_{k \in V} K\left(g_{k}, \varepsilon^{2}\right) d g_{k}
$$


The following proposition is a direct consequence of the classical Kolmogoroff theorem.

Proposition 1. There exists a unique probabilistic, Radon measure $\mu_{\varepsilon}$ on $\mathscr{G}_{\varepsilon}$ such that

$$
\left.\mu_{\varepsilon}\right|_{\mathscr{G}_{V}^{\varepsilon}}=\mu_{V}^{\varepsilon} .
$$

From now on we specify $\varepsilon=\frac{1}{2^{n}}$ and denote $\mathscr{G}_{\varepsilon}=\mathscr{G}_{n}$. According to Proposition 1 we have a probabilistic Radon measure $\mu_{n}$ on each $\mathscr{G}_{n}$ given by the heat kernels. Moreover there are natural maps $p_{n}: B \rightarrow \mathscr{G}_{n}$ given by the parallel transports:

$$
p_{n}(F)(k)=P_{c_{n, k}}(F) \text {, }
$$
where $k \in \frac{1}{2^{n}} Z^{2}$, and $C_{n, k}$ is a square of side length $\frac{1}{2^{n}}$, and the left, bottom corner in

Put the Wilson topology on $B$. Then we have

$-p_{n}$ are continuous

- $p_{n}$ separate points of $B$ (compare Lemma 3).

What we need now is to "pull-back" measures $\mu_{n}$ by $p_{n}$ to obtain a measure of Theorem 1. This would be done if we construct a projective system of measures and verify the Prokhorov Condition.

Lemma 5. There are unique continuous maps $p_{n, n+1}: \mathscr{G}_{n+1} \rightarrow \mathscr{G}_{n}$ such that

$$
\begin{aligned}
& p_{n, n+1} \cdot p_{n+1}=p_{n}, \\
& p_{n, n+1} \cdot \mu_{n+1}=\mu_{n} .
\end{aligned}
$$

(Recall that we work in the axial gauge.)

The reason that such maps actually exist is essentially equivalent to the possibility of computing integrals of Wilson loops by the usual lattice gauge theory methods. In consequence, the family $\left(B, \mathscr{G}_{n}, \mu_{n}, p_{n}, p_{n, n+1}\right)$ form a projective system of measures (see the appendix). Thus we only need to verify the Prokhorov condition.

Let $K_{R}$ be the closed ball in $B$ with radius $R$ and center in the origin. Then Lemma 3 implies that $K_{R}$ is compact in W.T. We have to estimate

$$
\sup _{n} \mu_{n}\left(\mathscr{G}_{n}-p_{n} K_{R}\right)
$$

which is equal to

$$
\sup _{n, v} \mu_{n}^{v}\left(\mathscr{G}_{n}^{v}-p_{n}^{v} p_{n} K_{R}\right) .
$$

This leads us to deal with finite dimensional integrals if only we are able to characterize $p_{n}^{v} p_{n} K_{R}$.

Proposition 2. Let $\tilde{K}_{R}^{v} \subset \mathscr{G}_{n}^{v}$ be defined as follows:

with

$$
\tilde{K}_{R}^{v}=\prod_{k \in V} g_{k} e^{i t_{k} \sigma} g_{k}^{-1}
$$

$$
g_{k} \in G, \quad t_{k} \in \mathbb{R}^{1}, \quad \text { and } \quad \sum t_{k} t_{l} J(k, l) \leqq R^{2} .
$$

Here $\sigma$ is the third Pauli matrix (say). 
Then $\widetilde{K}_{R}^{v} \subset p_{n}^{v} p_{n} K_{R}$.

This proposition is a simple exercise on locally constant functions. But now, we have

$$
\sup _{n} \mu_{n}\left(\mathscr{G}_{n}-p_{n} K_{R}\right) \leqq \sup _{n, v} \mu_{n}^{v}\left(\mathscr{G}_{n}^{v}-\widetilde{K}_{R}^{v}\right)
$$

Expressed in terms of maximal torus generated by $\delta$, the last expression becomes

$$
\int_{\substack{\left|t_{k}\right| \leq \pi \\ \Sigma t_{k} t_{l} J(k, l) \geqq R^{2}}} d \hat{\mu}_{n}^{v}\left(t_{1}\right) \ldots d \hat{\mu}_{n}^{v}\left(t_{|V|}\right),
$$

with some computable $d \hat{\mu}_{n}^{v}(t)$. The point is that $d \hat{\mu}_{n}^{v}(t)$ is even since $e^{i t \sigma}$ and $e^{-i t \sigma}$ are conjugate and the heat kernel is conjugation invariant. Next, notice that the characteristic function of $\left\{t_{k}: \sum t_{k} t_{l} J(k, l) \geqq R^{2}\right\}$ is smaller than the function $\left(t_{k}\right) \rightarrow \frac{1}{R^{2}} \sum t_{k} t_{l} J(k, l)$. Thus

$$
\mu_{n}^{v}\left(\mathscr{G}_{n}^{v}-\widetilde{K}_{R}^{v}\right) \leqq \int_{\left|t_{k}\right| \leqq \pi} \frac{1}{R^{2}} \sum_{k} J(k, k) t_{k}^{2} d \hat{\mu}_{n}^{v}\left(t_{1}\right) \ldots d \hat{\mu}_{n}^{v}\left(t_{|V|}\right),
$$

where non-diagonal terms drop out by evenness.

Estimating

$$
t^{2} \leqq C(2-2 \cos t) \quad \text { fr } \quad \| t \mid \leqq \pi \text { and some positive constant } C \text {, we can return }
$$
to the original group variables,

$$
\begin{aligned}
\mu_{n}^{v}\left(\mathscr{G}_{n}^{v}-\widetilde{K}_{R}^{v}\right) & \leqq \text { const } \frac{1}{R^{2}} \sum_{k} J(k, k) \int\left(2-\operatorname{Tr} g_{k}\right) d \mu_{n}^{v}\left(g_{k}\right) \\
& =\operatorname{const} \frac{1}{R^{2}} \sum_{k} J(k, k)\left\langle 2-\operatorname{Tr} P_{C_{n, k}}\right\rangle_{n, v}^{\text {lattice }} \\
& =\operatorname{const} \frac{1}{R^{2}} \sum_{k} J(k, k)\left(2-2 e^{-\frac{3}{4} \varepsilon^{2}}\right) \\
& \leqq \text { const } \frac{1}{R^{2}} \sum_{k} J(k, k) \varepsilon^{2}
\end{aligned}
$$

Now the Prokhorov condition is satisfied because $J$ is of trace class. Then the Prokhorov theorem guarantees the existence and uniqueness of the desired measure $\mu$ which is Radon in W.T. on B. Theorem A2 of the appendix finishes the proof since $B$ is a Polish space and we can uniquely extend $\mu$ to be Radon in the original topology too.

Our second main result is concerned with the functional measure for (QCD) after integrating out fermions. Recall that we have already defined the regularized determinants of the Dirac operator involved

$$
\operatorname{det}_{\varepsilon}^{v} \not D_{F}=\text { polynom. }\left(\operatorname{Tr} P_{c}(F)\right) \quad[\text { formula }(8)] \text {. }
$$

This expression may be extended to all $F \in B$ since $P_{C}$ can be extended to $B$ and $\operatorname{det}_{\varepsilon}^{v} \not D_{F}$ is a polynomial. 
Proposition 3. Functions $B \ni F \rightarrow \operatorname{det}_{\varepsilon}^{v} \not D_{F} \in \mathbb{R}^{1}$ are positive, continuous and integrable with respect to $\mu$.

This proposition enables us to define the sequence of measures

$$
d \tilde{\mu}_{\varepsilon}^{v}=\operatorname{det}_{\varepsilon}^{v} \not D_{F} d \mu(F) / \int \operatorname{det}_{\varepsilon}^{v} \not D_{F} d \mu(F) .
$$

The following theorem states the existence of the functional measure for (QCD) 2 defined as a suitable limit of measures $\tilde{\mu}_{\varepsilon}^{v}$ (for $V$ rectangular intersecting the $y$-axis).

Theorem 2. The sequence $\left\{\tilde{\mu}_{\varepsilon}^{v}\right\}$ is a weakly compact set is the space of probabilistic, Radon measures on $B$, where $B$ is equipped with the Wilson topology.

Remarks. 1. The space $B$ in the W.T. is not a completely regular space. We need a subtle weak topology on the space of measures on $B$ (see the appendix).

2. Any condensation point of $\left\{\tilde{\mu}_{\varepsilon}^{v}\right\}$ is a Radon measure also with respect to the original topology on $B$. It is a consequence of Theorem A2.

Idea of Proof. We would like to use the Prokhorov-Schwartz-Topsoe theorem (Theorem A4). Thus we need to estimate

$$
\sup _{\varepsilon, v} \tilde{\mu}_{\varepsilon}^{v}\left(B-K_{R}\right)
$$

This can be reduced to finite dimensional integrals because

$$
\operatorname{det}_{\varepsilon}^{v} \not D_{F}=\operatorname{det}_{\varepsilon}^{v} \circ p_{\varepsilon}^{v} \circ p_{\varepsilon}(F) .
$$

Here $\operatorname{det}_{\varepsilon}^{v}: \mathscr{G}_{\varepsilon}^{v} \rightarrow \mathbb{R}^{1}$ are uniquely determined by the formula (22). The notation is borrowed from the proof of Theorem 1.

Since we know the projections of $\mu$ by $p_{\varepsilon}^{v} p_{\varepsilon}$, we obtain (Proposition 2)

$$
\tilde{\mu}_{\varepsilon}^{v}\left(B-K_{R}\right) \leqq \frac{\int_{\mathscr{G}_{\varepsilon}^{v}-\tilde{K}_{R}^{v}} \operatorname{det}_{\varepsilon}^{v}(g) d \mu_{\varepsilon}^{v}(g)}{\int_{\mathscr{G}_{\varepsilon}^{v}} \operatorname{det}_{\varepsilon}^{v}(g) d \mu_{\varepsilon}^{v}(g)} .
$$

Now we intend to repeat the steps of the analogous estimations of Theorem 1, i.e.

- reduce integrals to the maximal torus

- estimate the characteristic function of $\left(\mathscr{G}_{\varepsilon}^{v}-\widetilde{K}_{R}^{v}\right)$ by $\frac{1}{R^{2}} \sum t_{k} t J(k, l)$ and $t^{2} \leqq$ const $(2-2 \cos t)$ [compare (18), (19)]

- return to the integral over $\mathscr{G}_{\varepsilon}^{v}$.

Everything would work but for the fact that $\operatorname{det}_{\varepsilon}^{v}$ is not conjugation invariant. This however can be easily overcome by the following, simple, group-theoretic lemma.

Lemma 6. Let $\mu$ be a conjugation invariant measure on a compact group $G$ and $f: G \rightarrow \mathbb{R}^{1}$ a continuous function on $G$. Then there exists a unique conjugation invariant function $\hat{f}: G \rightarrow \mathbb{R}^{1}$ such that

$$
\int_{A} f(g) d \mu(g)=\int_{A} \hat{f}(g) d \mu(g)
$$

for all conjugation invariant subsets $A \subset G$; in fact

$$
\hat{f}(g)=\int f\left(h g h^{-1}\right) d h,
$$

where $d h$ is the Haar measure on $G$. 
Lemma 6 can be directly applied in our situation since $\widetilde{K}_{R}^{v}$ and $d \mu_{\varepsilon}^{v}$ are conjugation invariant. Notice also, that by (23),

$$
\left[\left(2-\operatorname{Trg}_{k}\right) \operatorname{det}_{\varepsilon}^{v}\right]^{\wedge}=\left(2-\operatorname{Trg}_{k}\right)\left[\operatorname{det}_{\varepsilon}^{v}\right]^{\wedge} .
$$

Finally $\tilde{\mu}_{\varepsilon}^{v}\left(B-K_{R}\right)$, exactly as in $(20)$, is going to be less than

$$
\text { const } \frac{1}{R^{2}} \sum J(k, k)\left\langle 2-\operatorname{Tr} P_{C_{k}}\right\rangle_{\varepsilon, v}^{\text {lattice QCD }} .
$$

Let us underline that up to now our considerations were completely general, i.e. independent on particular form of the fermionic determinant. All the information we need is the following lattice QCD estimate:

$$
\left\langle 2-\operatorname{Tr} P_{C_{k}}\right\rangle_{\varepsilon, v}^{\text {lattice QCD }} \leqq \text { const } \varepsilon^{2}
$$

with constant independent on the volume and the location of plaquette $C_{k}$. The above estimate is presumably known to specialists, but since we have not found an exact reference, we give a proof of (24) in Appendix II.

This finishes the proof since $J$ is of trace class.

We end this section with some remarks and speculations.

1. Our methods cover two even simpler cases: Quantum Electrodynamics and Scalar Chromodynamics.

2. Presented results can be easily generalized to other groups like $S U(n)$ and to theories with few generations of fermions as well.

3. One may also consider applying the presented ideas to 3-dim Yang-Mills theory. Since in that case renormalization is necessary, one must add one more crucial technical ingredient: the renormalization group. Intuitively, the projective system will close provided $\mu_{\varepsilon}^{v}$ are fixed points of the R.G. Inequalities on $\left\langle 2-\operatorname{Tr} P \mathcal{C}_{k}\right\rangle_{\varepsilon, v}^{\text {lattice }}$ will control the dependence of the coupling constant on $\varepsilon$. Notice also that the (extended) axial gauge provides a natural way of defining the block spin transformation. We plan to discuss these questions in the near future.

4. We can prove the convergence of the regularized fermionic measures under very general assumptions. One can speculate that the same strategy can be applied in 3-dim, basically because the fermionic determinant does not need non-trivial renormalization in this case.

\section{Appendix A}

Throughout this appendix all topological spaces (t.s.) are assumed to be Haussdorf and all measures Borel and probabilistic. Basic references are $[7,8]$.

Definition A1. A measure $\mu$ on a t.s. $X$ is called a Radon measure iff for each Borel subset $A \subset X$,

$$
\mu(A)=\sup _{\substack{K \subset A \\ K \text { compact }}} \mu(K)
$$

Definition A2. $A$ t.s. $X$ is called a Polish space iff $X$ is homeomorphic to a metrizable, complete and separable t.s. 
Theorem A1. Any measure on a Polish space is a Radon measure.

Theorem A2. Let $X$ be a Polish space and let $f: X \rightarrow Y$ be a continuous bijection. Then $f$ induces a bijection between the spaces of Radon measures on $X$ and $Y$.

Corollary. Let $X$ be a Polish space and let $X_{w}$ be the set $X$ equipped with weaker topology. Then any Radon measure on $X_{w}$ can be uniquely extended to $X$.

(Take $f=\mathrm{id}: X \rightarrow X_{w}$ and use Theorem A2.)

The main tool in constructing Radon measures in infinite dimensions is the following theorem due to Prokhorov:

Theorem A3 (Prokhorov). Let $X, X_{i}$ be t.s. $-s$,

$\{i\}$ directed set of indices,

$p_{i}: X \rightarrow X_{i}$ continuous maps separating points of $X$,

$\mu_{i}-$ Radon measures on $X_{i}$.

Suppose that there exist maps $p_{i j}: X_{i} \rightarrow X_{j}$ for $i \geqq j$ such that

$$
p_{i j} p_{i}=p_{j}, \quad p_{i j} \cdot \mu_{i}=\mu_{j} .
$$

Then there exists a unique Radon measure $\mu$ on $X$ such that $p_{i} \cdot \mu=\mu_{i}$ iff for each $\varepsilon>0$ there exists a compact subset $K$ of $X$ such that for each $i$

$$
\mu_{i}\left(X_{i}-p_{i}(K)\right) \leqq \varepsilon \quad \text { (Prokhorov condition) }
$$

Definition $A 3$. A sequence $\left\{\mu_{i}\right\}$ of Radon measures on a t.s. $X$ converges weakly to $\mu$ iff

$$
\liminf _{j} \mu_{j}(f) \geqq \mu(f)
$$

for every bounded lower semicontinuous function $f$ on $X$.

If $X$ is a completely regular t.s. then the weak convergence of Definition A3 coincides with usual weak convergence

Theorem A4 (Prokhorov, Schwartz, Topsoe). Let $A$ be a set of Radon measures on a t.s. $X$ satisfying the following condition: for each $\varepsilon>0$ there exists $K \subset X$, $K$-compact, such that for each $\mu \in A: \mu(X-K) \leqq \varepsilon$. Then $A$ is relatively ( sequentially) compact in the weak topology of Definition $A 3$.

\section{Appendix B}

In this appendix we give a proof of inequality (24).

Basic references are $[5,9]$.

Let $H_{v}$ be the space of functions on $v \subset \varepsilon \mathbb{Z}^{2}$ taking values in $\mathbb{C}^{2} \otimes \mathbb{C}^{2}$. Equip $H_{v}$ with the scalar product

$$
(f, g)=\sum_{x \in v} \sum_{i} \bar{f}^{i}(x) g^{i}(x)=\sum_{x \in v} \bar{f}(x) g(x) .
$$

(In what follows we shall consequently omit upper indices.) Canonical matrix elements of linear operators in $H_{v}$ are given by

$$
A f(x)=\sum_{y} A(x, y) f(y) .
$$


The Dirac operator we are interested in has the following matrix elements:

$$
\not_{g}(x, y)=\left(\frac{2}{\varepsilon}+m\right) \delta_{(x, y)}-\frac{1}{\varepsilon} g(x, y) \gamma(x, y),
$$

where the last term is non-vanishing for the nearest neighbours only,

$$
\begin{gathered}
g(x, y) \in S U(2), \quad g(y, x)=g(x, y)^{-1}, \\
\gamma\left(x, x \pm e_{\mu}\right)=\frac{1 \mp \sigma_{\mu}}{2},
\end{gathered}
$$

and $\sigma_{0}, \sigma_{1}$ are Pauli matrices.

Let us single out plaquette $\bar{p} \subset V$ and define operator $\not_{g}^{0}$ by

$$
D_{g}^{0}(x, y)= \begin{cases}\not D_{g}(x, y) & \text { for } \quad(x, y) \notin \bar{p} \\ \left(\frac{2}{\varepsilon}+m\right) \delta(x, y)-\frac{1}{\varepsilon} \gamma(x, y) & \text { for } \quad(x, y) \in \bar{p}\end{cases}
$$

\section{Lemma.}

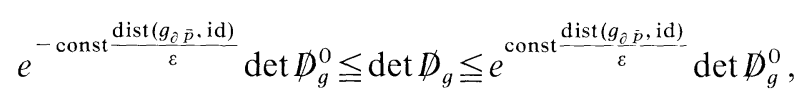

where $g_{\partial \bar{p}}$ is a product of $g_{(x, y)}$ along $\bar{p}$ and $\operatorname{dist}(\cdot, \cdot)$ is a distance with respect to the Killing metric on $S U(2)$.

Proof. Notice that $\operatorname{det} D_{g}$ is lattice gauge invariant, i.e. invariant under transformations

$$
g(x, y) \rightarrow h(x) g(x, y) h(y)^{-1}, \quad h(x) \in S U(2) .
$$

Thus without loss of generality we can put $g(x, y)=\mathrm{id}$ on three bonds of $\bar{p}$. On the remaining bond $\bar{b} \in \bar{p}$ we shall have $g_{\bar{b}}=g_{\hat{\partial} \bar{p}}$. Consider the one parameter family of operators $D_{t}$ obtained from $\not_{g}$ by replacing $g_{\bar{b}} \rightarrow g_{b}^{t}$, where $g_{b}^{t}$ is a minimal geodesic joining id and $g_{\bar{b}}$ in a unit time. Thus

We have

$$
\operatorname{det} D_{0}=\operatorname{det} \not D_{g}^{0} ; \quad \operatorname{det} D_{1}=\operatorname{det} \not \emptyset_{g} .
$$

$$
\begin{aligned}
\left|\log \left(\operatorname{det} \not D_{g} / \operatorname{det} \not D_{g}^{0}\right)\right| & =\left|\int_{0}^{1} \frac{d}{d t}\left(\log \operatorname{det} D_{t}\right) d t\right| \\
\leqq & \int_{0}^{1} d t\left|\operatorname{Tr} \dot{D}_{t} D_{t}^{-1}\right| \leqq \operatorname{const} \int_{0}^{1}\left\|\dot{D}_{t}\right\|\left\|D_{t}^{-1}\right\| d t .
\end{aligned}
$$

Here $\|\cdot\|$ is the operator in the space of operators on $H_{V}$. Observe that const is volume independent since $\dot{D}_{t}$ has only two non-vanishing matrix elements. It is easy to see that

Moreover

$$
\left\|\dot{D}_{t}\right\|=\frac{\operatorname{dist}\left(g_{\partial \bar{p}}, \text { id }\right)}{\varepsilon} .
$$

$$
\left\|D_{t}^{-1}\right\| \leqq \frac{1}{m} \quad(\text { see }[9, \text { Corollary } 3.2])
$$

(we always assume that the mass $m$ is strictly positive). Putting together (B6), (B7), and (B8), we get (B5). 
We would like to estimate

where

$$
\left\langle 2-\operatorname{Tr} g_{\partial \bar{p}}\right\rangle_{\varepsilon, v}^{\text {lattice QCD }}=N^{-1} \int \prod_{b} d g_{b}\left(2-\operatorname{Trg}_{\partial \bar{p}}\right) \operatorname{det} \not D_{g} \prod_{p} K\left(g_{\partial \bar{p}}, \varepsilon^{2}\right),
$$

$$
N=\int \prod_{b} d g_{b} \operatorname{det} \not D_{g} \prod_{p} K\left(g_{\partial p}, \varepsilon^{2}\right)
$$

By the lemma this is smaller than

$$
N^{-1} \int \prod_{b} d g_{b}\left(2-\operatorname{Tr} g_{\hat{\partial} \bar{p}}\right) \operatorname{det} \not D_{g}^{0} e^{\operatorname{const} \frac{\operatorname{dist}\left(g_{\hat{\partial} \bar{p}}, \mathrm{id}\right)}{\varepsilon}} \prod_{p} K\left(g_{\partial p}, \varepsilon^{2}\right)
$$

with $N$ equal now

$$
N=\int \prod_{b} d g_{b} \operatorname{det} \not D_{g}^{0} e^{-\operatorname{const} \frac{\operatorname{dist}\left(g_{\partial \dot{p}}, \text { id }\right)}{\varepsilon}} \prod_{p} K\left(g_{\partial p}, \varepsilon^{2}\right) .
$$

To simplify the notation we shall denote by $N$ any denominator in formulas we get and by const any numerical constant. These objects vary from case to case in an obvious manner so we shall not write them down explicitly.

In (B12) the integral over $\bar{p}$ separates from the rest by independence of Wilson loops. Cancelling between the numerator and the denominator, we have to estimate single integrals only

$$
\left\langle 2-\operatorname{Tr} g_{\hat{o} \bar{p}}\right\rangle_{\varepsilon, v}^{\mathrm{QCD}} \leqq N^{-1} \int d g(2-\operatorname{Tr} g) e^{\mathrm{const}-\frac{\sqrt{2-\frac{\operatorname{Tr}}{\varepsilon}}}{\varepsilon}} K\left(g, \varepsilon^{2}\right)
$$

where we have used (19),

$$
\operatorname{dist}^{2}(g, i d) \leqq \operatorname{const}(2-\operatorname{Tr} g) .
$$

More direct form of the right-hand side of (B13) may be obtained by passing to the maximal torus. Using the adequate formula for the heat kernel [5, formula (9)], we get

$$
N^{-1} \prod_{-\pi}^{\pi} d \phi \sin ^{2} \frac{\phi}{2} e^{\mathrm{const}-\frac{\sin \frac{\phi}{2} \mid}{\varepsilon}} \sum_{n=-\infty}^{\infty}(\phi+2 \pi n) \sin \phi e^{-\frac{(\phi+2 \pi n)^{2}}{\varepsilon^{2}}} .
$$

Changing variables in each term of the sum and using periodicity of the integrand one can express (B14) as integrals over $\mathbb{R}^{1}$. We state the formula obtained after rescaling $d \rightarrow \frac{d}{\varepsilon}$ and suitable rearranging,

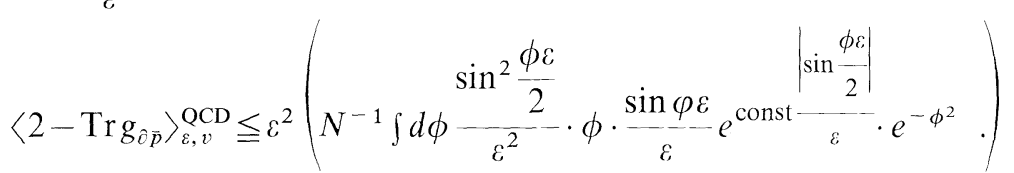

By the Lebesgue dominant convergence theorem, the expression in parenthesis is a continuous function of $\varepsilon$ for $\varepsilon>0$ with the limit, as $\varepsilon$ goes to 0 , equal to

$$
\text { const } \int \phi^{4} e^{\text {const }|\phi|} e^{-\phi^{2}} d \phi / \int \phi^{2} e^{- \text {const }|\phi|} e^{-\phi^{2}} d \phi \text {. }
$$

This proves the inequality (24) (for sufficiently small $\varepsilon$ ). 


\section{References}

1. Klimek, S.: Warsaw University Ph. D. Thesis (in preparation)

2. King, C.: The $U(1)$ Higgs model. I. The continuum limit. Commun. Math. Phys. 102, 649-678 (1986)

3. Singer, I.M.: The geometry of the orbit space for non-abelian gauge theories. Phys. Scripta 24, $817-820(1981)$

4. Seiler, E.: Gauge theories as a problem of constructive quantum field theory and statistical mechanics. Berlin, Heidelberg, New York: Springer 1982

5. Menotti, P., Onofri, E.: The action of $S U(N)$ lattice gauge theory in terms of the heat kernel on the group manifold. Nucl. Phys. B 190 (FS3), 288-301 (1981)

6. Stamatescu, I.O.: Note on the lattice fermionic determinant. Phys. Rev. D 25, 1130-1135 (1982)

7. Bourbaki, N.: Elements de mathematique-Integration. Paris: Hermann 1971

8. Schwartz, L.: Radon measures on arbitrary topological spaces and cylindrical measures. Bombay: Oxford 1973

9. Weingarten, D.H., Challifour, J.L.: Continuum limit of $(\mathrm{QED})_{2}$ on a lattice. Ann. Phys. 123, 61-101 (1979)

Communicated by K. Gawedzki

Received February 18, 1987; in revised form June 1, 1987 